\section{Bestrahlung oder Operation beim NSCLC?}

Beim nichtkleinzelligen Lungenkarzinom (NSCLC) im Stadium I gilt die Lobektomie als Behandlung der Wahl. Zunehmend wird jedoch auch stereotaktisch bestrahlt - selbst bei operablen Patienten. Ist das eine gute Alternative?

ie SBRT (Stereotactic Body Radiation Therapy) soll möglichst wenig Strahlung hochpräzise auf extrakraniale Tumoren geben. Bislang ist der Standard beim NSCLC im Stadium I die Lobektomie mit mediastinaler Lymphknoten- dissektion - außer bei Patienten mit schwerer Komorbidität oder Patienten, die ohnehin weniger invasive Prozeduren bevorzugen. Für sie könnte die SBRT eine neue Option sein. Die Japan Clinical Oncology Group (JCOG) überprüfte

\begin{tabular}{l|l|l}
$\begin{array}{l}\text { Tab. 1: Muster des Therapieversagens (inoperabel und operabel) } \\
\text { Muster }\end{array}$ & inoperabel & operabel \\
\hline $\begin{array}{l}\text { Lokal }(\mathrm{n}=20) \\
\text { Lokal + Lymphknotenbefall }\end{array}$ & 6 & 1 \\
\hline Lokal + Fernmetastasierung & 1 & 1 \\
\hline Lokal + Lymphknotenbefall + Fernmetastasierung & 4 & 4 \\
\hline Lymphknotenbefall ( $\mathrm{n}=24)$ & 0 & 3 \\
\hline Lymphknotenbefall + Fernmetastasierung & 1 & 4 \\
\hline Fernmetastasierung $(\mathrm{n}=44)$ & 6 & 8 \\
\hline Gesamt & 13 & 6 \\
\hline & 31 & 27
\end{tabular}

\section{Phase-III-Studie mit Tivantinib bei NSCLC}

Die Inhibition des c-Met-Signalwegs hat zuletzt Hoffnung gemacht. Beim nichtkleinzelligen Lungenkarzinom (NSCLC) vom Nicht-Plattenepithel-Typ konnte der experimentelle Inhibitor Tivantinib in einer Phase-II-Studie das progressionsfreie Überleben verlängern. Nun ging die Prüfung in die Phase III.

$\mathrm{N}$ eben dem Rezeptor für epidermalen Wachstumsfaktor (EGFR) und dem vaskulären endothelialen Wachstumsfaktor (VEGF) bieten sich weitere Stoffwechselwege als Angriffspunkte für die NSCLC-Therapie an. Vor kurzem wurde in einer randomisierten Phase-II-Studie gezeigt, dass die Zugabe des experimentellen c-Met-Inhibitors Tivantinib zum EGFR-Inhibitor Erlotinib beim vorbehandelten Nicht-Plattenepithelkarzinom der Lunge das progressionsfreie Überleben (PFS) verlängern könnte. In einer Subgruppenanalyse war der Überlebensvorteil bei Patienten mit Wildtyp(WT)EGFR größer als bei solchen mit aktivie-

renden EGFR-Mutationen. In einer Phase-III-Studie erhielten deshalb asiatische Patienten mit Nicht-Plattenepithel-NSCLC und WT-EGFR Erlotinib plus Tivantinib oder plus Placebo. Ursprünglich sollten 460 Patienten randomisiert werden, primärer Endpunkt war das Gesamtüberleben, sekundäre Endpunkte PFS, Ansprechen und Sicherheit.

Nach Aufnahme von 307 Patienten wurde die Studie gestoppt, weil im Tivantinib-Arm 14 Patienten eine interstitielle Lungenkrankheit (ILD) entwickelt hatten; 3 verstarben. Im Placeboarm gab es 6 Fälle von ILD und keinen Todesfall. Das mediane Gesamtüberle-
Sicherheit und Wirksamkeit bei 164 auswertbaren Patienten mit NSCLC im Stadium T1 N0 M0 (100 operabel, 64 inoperabel). Diese erhielten 48 Gy in 4 Fraktionen. Primärer Endpunkt war die Rate für das 3-Jahres-Gesamtüberleben, das bei den inoperablen Patienten mindestens $35 \%$, bei den operablen mindestens $80 \%$ betragen sollte.

Diese Vorgaben wurden bei den inoperablen Patienten mit 59,9\% weit übertroffen, Toxizitäten vom Grad 3, 4 oder 5 traten bei 10, 2 bzw. keinem Patienten auf. Von den 64 operablen Patienten lebten nach 3 Jahren noch 76,5\%, lediglich Nebenwirkungen vom Grad 3 kamen bei 5 Patienten vor.

Fazit: Die SBRT ist beim NSCLC im Stadium I effektiv und wenig toxisch. Sie kann als Standard für das inoperable NSCLC im Stadium I gelten. Auch bei operablen Tumoren ist sie eine vielversprechende Alternative. Christina Berndt

Nagata $Y$ et al. Prospective Trial of Stereotactic Body Radiation Therapy for Both Operable and Inoperable T1NOMO Non-Small Cell Lung Cancer: Japan Clinical Oncology Group Study JCOG0403. Int J Radiat Oncol Biol Phys. 2015; 93(5):989-96.

ben lag unter Tivantinib bei 12,7 , unter Placebo bei 11,1 Monaten (Hazard Ratio [HR] 0,891; $\mathrm{p}=0,427)$, das mediane PFS betrug 2,9 bzw. 2,0 Monaten (HR 0,719; $\mathrm{p}=0,019)$. Häufigste Nebenwirkungen vom Grad $\geq 3$ unter Tivantinib waren Neutropenie (24,3\%), Leukopenie $(18,4 \%)$, febrile Neutropenie (13,8\%) und Anämie (13,2\%).
Fazit: Der vorzeitige Abbruch der Studie ging zu Lasten der statistischen Power. Es zeigte sich keine signifikante Verbesserung des Gesamtüberlebens durch Tivantinib. Allerdings könnte es das PFS von entsprechenden Patienten verbessern.

Yoshioka $\mathrm{H}$ et al. A randomized, double-blind, placebo-controlled, phase III trial of erlotinib with or without a c-Met inhibitor tivantinib (ARQ 197) in Asian patients with previously treated stage IIIB/IV nonsquamous nonsmallcell lung cancer harboring wild-type epidermal growth factor receptor (ATTENTION study). Ann Oncol. 2015;26(10):2066-72.
Christina Berndt 